\title{
Using Patient-Derived Xenografts to Explore the Efficacy of Treating Head-and-Neck Squamous Cell Carcinoma With Anlotinib
}

\begin{abstract}
Fangling $\mathrm{Hu}^{1}$, Liang Guo ${ }^{1}$, Jieqing $\mathrm{Yu}^{2}$, Daofeng Dai ${ }^{1,2}$, Yuanping Xiong ${ }^{1,2 *}$, Yuanqiao $\mathrm{He}^{3 *}$ and Wensheng Zhou ${ }^{1,2 *}$

${ }^{1}$ Department of Otolaryngology-Head and Neck Surgery, First Affiliated Hospital of Nangchang University, Nanchang, China, 2 Jiangxi Institute of Otorhinolaryngology-Head and Neck Surgery, Nanchang, China, ${ }^{3}$ Laboratory Animal Science Center of Nanchang University, Nanchang, China
\end{abstract}

Objective: The efficacy of anlotinib as a treatment for head-and-neck squamous cell carcinoma (HNSCC) has been little explored. Here, we used patient-derived xenografts (PDXs) to this end.

Methods: Fresh tumor tissues of HNSCC patients were screened in terms of in vitro drug sensitivity using the MTT assay. Patient PDXs were used to confirm the anti-tumor effects of anlotinib in vivo. After the medication regimen was complete, the tumor volume changes in mice were calculated. Apoptosis was measured using the TUNEL assay. The cell proliferation and apoptosis levels of PDXs yielded data on the utility of anlotinib treatment in vivo.

OPEN ACCESS

Edited by:

Andrea Ladányi,

National Institute of Oncology (NIO),

Hungary

*Correspondence:

Yuanping Xiong

xiongyp@ncu.edu.cn

Yuanqiao $\mathrm{He}$

heyuanqiao@ncu.edu.cn

Wensheng Zhou

zhouwesh@163.com

Received: 05 August 2021 Accepted: 10 November 2021 Published: 09 December 2021

Citation:

Hu F, Guo L, Yu J, Dai D, Xiong Y, He Y and Zhou W (2021) Using Patient-

Derived Xenografts to Explore the Efficacy of Treating Head-and-Neck

Squamous Cell Carcinoma

With Anlotinib.

Pathol. Oncol. Res. 27:1610008.

doi: 10.3389/pore.2021.1610008
Results: Anlotinib suppressed the in vitro proliferation of nine tumor tissues by an average of $51.05 \pm 13.74 \%$. Anlotinib also significantly inhibited the growth of three PDXs in mice (tumor growth inhibition 79.02\%). The expression levels of Ki-67 and proliferating cell nuclear antigen after anlotinib treatment were significantly lower than those in the controls. The negative and positive controls exhibited no and some apoptosis, respectively, whereas the anlotinib group evidenced extensive apoptosis.

Conclusion: Anlotinib suppressed HNSCC growth in vitro and in vivo (by inhibiting cell proliferation and promoting apoptosis), suggesting that anlotinib can potentially treat HNSCC.

Keywords: head-and-neck squamous cell carcinoma, drug sensitivity, patient-derived xenograft, anlotinib, tumor inhibition

\section{INTRODUCTION}

Head-and-neck cancers arise from the mucosal surfaces of the oral and sinonasal cavities, oropharynx, hypopharynx, and larynx. Such cancers constitute the seventh most common cancer group worldwide (3\% of all malignancies in 2018) (1). Over $90 \%$ of such cancers are head-and-neck squamous cell carcinomas (HNSCCs), with over 890,000 new cases and 450,000 deaths reported annually in the United States $(1,2)$. There are several risk factors, of which smoking and alcohol consumption are the most common $(3,4)$. Recently, human papillomavirus has been 
confirmed to contribute to oropharyngeal carcinoma development, especially in young males (5). Although surgery, radiation therapy, chemotherapy, and combination therapy have been used to treat HNSCC for decades, the prognosis remains unsatisfactory $(6,7)$.

Angiogenesis plays a key role in the creation, development, and metastasis of solid tumors. Therefore, anti-tumor angiogenesis therapies have become popular. Vascular endothelial growth factor (VEGF) is key in terms of tumor angiogenesis. VEGF triggers downstream signaling by activating three structurally related VEGF receptor (VEGFR) tyrosine kinases (VEGFR1, VEGFR2, and VEGFR3), thus promoting tumor angiogenesis and metastasis (8-13).

Anlotinib is a very potent, multi-targeting, oral tyrosine kinase inhibitor that blocks VEGFR2 phosphorylation as well as the actions of platelet-derived growth factor receptor $\alpha / \beta$ (PDGFR $\alpha / \beta)$, c-Kit, Ret, Aurora-B, c-FMS, and the discoid region of domain receptor 1 (DDR1) $(14,15)$. Anlotinib also inhibits tumor angiogenesis induced by VEGF, PDGF, and FGF-2 (16, 17). Phase II/III clinical trials revealed that anlotinib had encouraging effects in patients with a variety of solid tumors, including non-smallcell lung cancers, hepatocarcinomas, renal carcinomas, gastric cancers, and soft tissue sarcomas (15). The efficacy of anlotinib in patients with advanced lung squamous cell carcinomas has been confirmed in clinical trials, and the drug is approved as the thirdline treatment for such cancers (18). We hypothesized that anlotinib would inhibit HNSCC growth, invasion, and metastasis and would usefully treat refractory advanced cancers. As no report on the potential anti-tumor effects of anlotinib on animal HNSCC has appeared, we collected fresh HNSCC samples and constructed patient-derived xenografts (PDXs) to explore the therapeutic effects of the drug.

\section{MATERIALS AND METHODS}

\section{In Vitro Drug Sensitivities}

All samples were collected at the First Affiliated Hospital of Nanchang University and all procedures were approved by the ethics committee of the hospital (approval no. 017). Informed patient consent was obtained before surgery. Nine HNSCCs were surgically removed, divided into $2 \times 2 \times 2 \mathrm{~mm}^{3}$ sections, and placed in 96-well plates (one block/well) at a low temperature. After injection of a water-containing gel, the tissues were cultured at $37^{\circ} \mathrm{C}$ at $95 \%$ humidity under $5 \%(\mathrm{v} / \mathrm{v}) \mathrm{CO}_{2}$. Three-quarters of the medium containing different drugs was changed every 1-2 days. The control cells were treated with Dulbecco's modified Eagle medium/F12 complete medium (Cat. No.11330107; Gibco, Amarillo, TX, United States) supplemented with $10 \%(\mathrm{v} / \mathrm{v})$ fetal bovine serum (Cat. No.16140063; Gibco), 100 units/ml penicillin, and $100 \mu \mathrm{g} / \mathrm{ml}$ streptomycin. The tissues in the control groups were cultured in a manner similar to control cells. The drug groups were cultured in complete medium with $3 \%(\mathrm{v} / \mathrm{v})$ dimethyl sulfoxide (DMSO, Cat. No. D4540; Sigma, St. Louis, MO, United States), $24 \mu \mathrm{M}$ anlotinib (Zhengda Tianqing Pharmaceutical Group Co. Ltd., Nanjing, China), $20 \mu \mathrm{M}$ sunitinib (Pfizer Pharmaceuticals Ltd., New York, NY, United States), $9 \mu \mathrm{M}$ apatinib (Hengrui Pharmaceuticals, Jiangsu, China), $75 \mu \mathrm{g} / \mathrm{ml}$ paclitaxel (Beijing SL Pharmaceutical Co. Ltd., Beijing, China), or $25 \mu \mathrm{g} / \mathrm{L}$ cisplatin (Harvey, United States). The culture medium was replaced after $24 \mathrm{~h}$ to maintain the activity and morphology of the original tissue block. On day 8 , the medium was removed, tissues were washed three times with phosphate-buffered saline, and MTT solution (Sigma) was added. After incubation for $16 \mathrm{~h}$, the tissue blocks were placed in new 96-well plates, and each block was incubated with $200 \mu \mathrm{l}$ DMSO for $6 \mathrm{~h}$. Aliquots of $100 \mu \mathrm{l}$ DMSO were then placed into the wells of a fresh 96-well plate, and the absorbance (A values) at $490 \mathrm{~nm}$ were measured. Drug sensitivity was assessed based on the tumor inhibition rate, which was calculated as $[1-$ (drug A-blank A/control A-blank A) $\times 100 \%$; a tumor inhibition rate $\geq 30 \%$ was presumed to indicate drug sensitivity. The experiment was repeated three times and averages were calculated.

\section{PDXs}

PDXs (numbered as P0) were established. Three PDX models, one of hypopharyngeal carcinoma and two of laryngeal carcinomas, were selected and passaged to P3 by the same method, then used for in vivo experiments. When the diameters of the third-generation subcutaneous tumors reached $1 \mathrm{~cm}$, they were minced $\left(2 \times 2 \times 2 \mathrm{~mm}^{3}\right)$ and subcutaneously implanted into the flanks of 4- to 6-week-old nude mice. Each PDX was inoculated into 24 mice. When the tumor volumes reached $100-200 \mathrm{~mm}^{3}$, the mice were randomly divided into three groups of six: a control group (normal saline $100 \mu \mathrm{l}$ intragastrically per day), a drug group (anlotinib $3 \mathrm{mg} / \mathrm{kg}$ intragastrically per day), and a positive control group (cisplatin $5 \mathrm{mg} / \mathrm{kg}$ intraperitoneally per week). Mouse health and tumor growth were observed daily. The tumor volume and mouse body weight were recorded every 4 days. Twenty-one days later, when the tumor volume in the control group reached $1,500 \mathrm{~mm}^{3}$, all mice were sacrificed via cervical dislocation, and the tumor tissues were harvested. Tumor size was measured using a caliper, and tumor volume was calculated as $1 / 2 \times$ length $\times(\text { width })^{2}$. Tumor growth inhibition (TGI) was used to evaluate the anti-tumor effects of anlotinib. TGI (\%) was calculated as: TGI (\%) = [1-(tumor volume at the end of the drug treatment group-tumor volume at the beginning of the drug treatment group)/(tumor volume at the end of the control group-tumor volume at the beginning of the control group)] $\times 100 \%$. All experiments were approved by the Animal Ethics Committee of Nanchang Leyou Biotechnology Co. Ltd.

PDX construction was performed using the protocol established by Karamboulas et al. (19). Briefly, HNSCC-PDXs were generated, the tumor tissues were processed, and the PDX models were expanded into cohorts for drug testing.

\section{Pathology}

Formalin-fixed PDX samples were embedded in paraffin and cut into $3-\mu \mathrm{m}$-thick sections that were baked at $60^{\circ} \mathrm{C}$ for $12 \mathrm{~h}$. The sections were then placed into xylene for 5-10 min (repeated three times), hydrated in $100,100,95,85$, and $75 \%(w / w)$ ethanol 
TABLE 1 | Drug inhibition of HNSCC tissue proliferation.

\begin{tabular}{|c|c|c|c|c|c|c|c|}
\hline \multirow[t]{2}{*}{ Sample number } & \multirow[t]{2}{*}{ Histological origin } & \multicolumn{6}{|c|}{ Tumor inhibition rate (\%) } \\
\hline & & DMSO & Paclitaxel & Cisplatin & Anlotinib & Sunitinib & Apatinib \\
\hline No. 3 & Oropharynx & $5.11 \pm 1.45$ & $44.92 \pm 1.80$ & $42.05 \pm 1.00$ & $44.57 \pm 0.76$ & $27.56 \pm 1.09$ & $35.62 \pm 0.90$ \\
\hline No. 4 & Oropharynx & $9.46 \pm 0.65$ & $55.26 \pm 0.64$ & $44.62 \pm 0.75$ & $51.64 \pm 0.72$ & $17.98 \pm 1.03$ & $38.09 \pm 0.71$ \\
\hline No. 7 & Larynx & $5.00 \pm 0.21$ & $44.44 \pm 0.06$ & $36.76 \pm 0.06$ & $32.41 \pm 0.43$ & $21.38 \pm 0.59$ & $1.63 \pm 0.50$ \\
\hline No. 8 & Larynx & $18.78 \pm 0.26$ & $46.65 \pm 0.37$ & $40.85 \pm 0.12$ & $70.29 \pm 0.10$ & $16.39 \pm 0.09$ & $13.18 \pm 0.15$ \\
\hline No. 10 & Hypopharynx & $6.20 \pm 0.41$ & $49.66 \pm 0.12$ & $54.51 \pm 0.10$ & $68.97 \pm 0.04$ & $24.63 \pm 0.04$ & $13.69 \pm 0.15$ \\
\hline No. 12 & Larynx & $9.30 \pm 0.72$ & $39.02 \pm 2.60$ & $43.11 \pm 0.41$ & $44.26 \pm 0.39$ & $36.11 \pm 0.53$ & $21.57 \pm 0.47$ \\
\hline No. 15 & Oropharynx & $5.54 \pm 0.28$ & $37.37 \pm 0.02$ & $24.74 \pm 0.15$ & $31.51 \pm 0.25$ & $34.52 \pm 0.13$ & $28.16 \pm 0.12$ \\
\hline No. 17 & Larynx & $24.95 \pm 0.27$ & $77.3 \pm 0.14$ & $36.55 \pm 0.22$ & $64.83 \pm 0.15$ & $61.46 \pm 0.21$ & $59.31 \pm 0.22$ \\
\hline No. 19 & Oropharynx & $13.51 \pm 0.07$ & $55.78 \pm 0.13$ & $53.74 \pm 0.22$ & $51.01 \pm 0.20$ & $30.21 \pm 0.10$ & $14.28 \pm 0.16$ \\
\hline
\end{tabular}

Absorbance (A) at $490 \mathrm{~nm}$ was used to calculate drug sensitivity as follows: tumor inhibition rate $=(1-$ drug A/control A) $\times 100 \%$; tumor inhibition rate $\geq 30 \%$ indicates drug sensitivity.

A

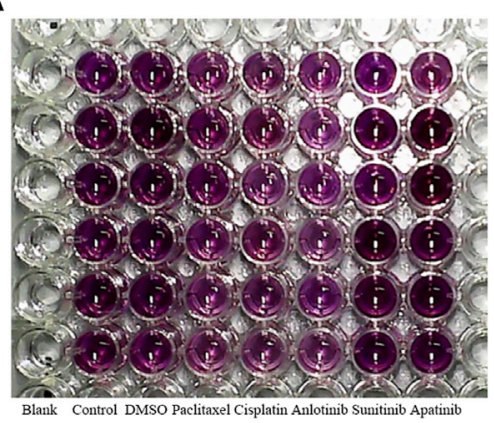

B

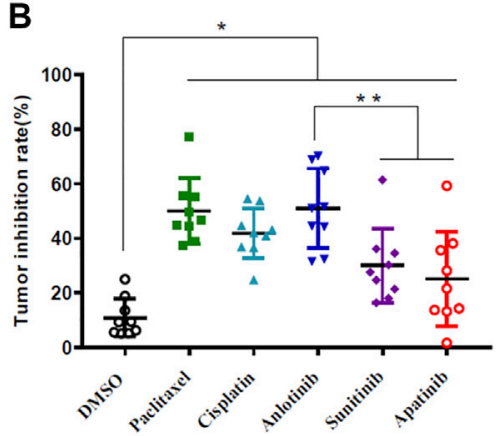

FIGURE 1 | (A) Representative results: 7-days drug sensitivity of HNSCC tissues measured using the MTT assay. (B) Quantitative data from three independent experiments are shown in the right panels. ${ }^{*} p<0.05 ;{ }^{* *} p<0.01$.

(in sequence) for $5 \mathrm{~min}$ each, soaked in distilled water for $5 \mathrm{~min}$, stained with hematoxylin (Wellbio, China) for 5-10 min, washed with distilled water, stained with eosin (Wellbio) for 3-5 min, washed with distilled water, dehydrated in 95 and 100\% (v/v) alcohol baths for $5 \mathrm{~min}$ each, placed in xylene for $10 \mathrm{~min}$ (two repeats), mounted with neutral gum, and observed microscopically.

\section{Terminal Deoxynucleotidyl Transferase-Mediated Deoxyuridine Triphosphate Nick-End Labeling Immunohistochemistry}

After dewaxing, some tissues were used for immunohistochemistry and TUNEL assays. Some slides were subjected to Proteinase $\mathrm{K}$ incubation and used for permeabilization; positive slides were incubated with recombinant terminal deoxynucleotidyl transferase enzyme, Equilibration Buffer, dUTP Labeling Mix, whereas negative slides were incubated with distilled water instead of recombinant terminal deoxynucleotidyl transferase enzyme. The TUNEL Apoptosis Detection Kit featuring fluorescein isothiocyanate (Cat. No. 40306ES20) was purchased from
Shanghai YEASEN (Shanghai, China). DAPI (Cat. No. 2871890-3) was obtained from Proteintech (Rosemont, IL, United States). Apoptosis was evaluated after drug treatment in vivo, following the manufacturer's instructions.

The TUNEL assay was performed as described by Loo (20). Briefly, tissues were fixed in formaldehyde and permeabilized with Proteinase $\mathrm{K}$ to allow the penetration of TUNEL reaction reagents into the cell nucleus. After fixation and washing, the incorporation of biotinylated dUTP onto the $3^{\prime}$ ends of fragmented DNA was conducted in a reaction containing terminal deoxynucleotidyl transferase. The slides were mounted with $90 \%$ glycerol and visualized by fluorescence microscopy. The TUNEL stained slides were calculated by manual counting under a fluorescent microscope. The TUNEL-stained cells appeared green, while the other cell nuclei appeared blue. The apoptosis index (AI) was regarded as the number of TUNEL-positive cells/total number of cells $\times 100 \%$.

\section{Immunohistochemistry}

Immunohistochemistry (IHC) was performed as previously described (21). Briefly, paraffin-embedded xenografts or tissues were cut into $3-\mu \mathrm{m}$ sections and mounted on slides, subjected to antigen retrieval in hot citrate buffer $(\mathrm{pH}=7.4)$, 

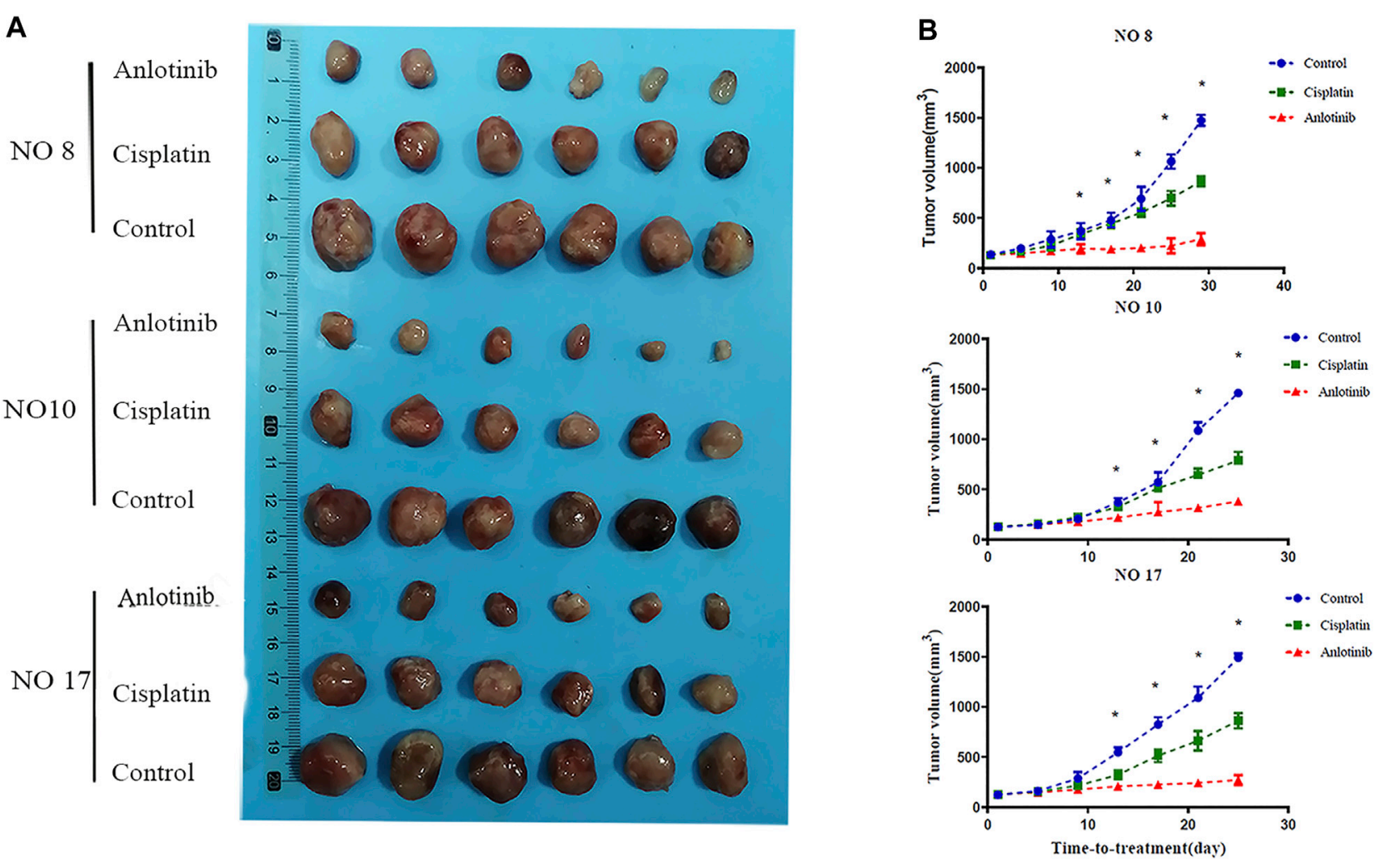

FIGURE 2 | Anti-tumor effects of anlotinib in the PDX model. (A) Representative tumor images after treatment. (B) Tumor volumes every 4 days. When the volume in the control group reached $1,500 \mathrm{~mm}^{3}$, all tumors were harvested.

then blocked in $10 \%$ normal goat serum. Subsequently, sections were incubated with primary antibodies against Ki-67 or proliferating cell nuclear antigen (Cat. Nos. 27309-1-AP and 10205-2-AP PCNA; Proteintech). The slides were incubated with goat anti-mouse/rabbit poly-horseradish peroxidaseconjugated secondary antibodies (Cat. No. PR30009; Proteintech). Nuclear Ki-67, as well as nuclear and cytoplasmic PCNA, stained red clay or brown. The PCNA slides were scored and analyzed using Image-Pro Plus software. Six 400X images were analyzed for each slide. The average integrated optical density was calculated as the ratio of the positive area to the total area. The Ki67 slides were analyzed using a microscope. Six 400X images were analyzed for each slide. The positivity cell rate was calculated as the ratio of the number of positive cells to the number of total cells.

\section{Statistical Analysis}

Statistical analyses were performed using the SPSS software package (ver. 22.0; IBM, United States). All graphs were produced using Graph Pad Prism ver. 7.0 for Windows (Graph Pad Software Inc., United States). All experiments were performed in triplicate, with the means of those three independent experiments calculated. Data are presented as the means \pm standard deviations (SDs) and were compared using Student's t-test (two groups) or one-way ANOVA (multiple groups). A $p$-value $<0.05$ was considered to indicate statistical significance.

\section{RESULTS}

\section{In Vitro Drug Sensitivities}

Tumor samples from nine HNSCC patients were cultured in vitro at $37^{\circ} \mathrm{C}$ under $5 \%(\mathrm{v} / \mathrm{v}) \mathrm{CO}_{2}$ (Table 1). The samples were labeled as Control, DMSO, anlotinib, sunitinib, apatinib, paclitaxel, and cisplatin. We used the MTT assay to assess tumor cell viability; anlotinib significantly inhibited cell viability (Figure 1A; Table 1). For all nine samples, anlotinib exhibited a greater inhibitory effect than did sunitinib and apatinib. The tumor inhibition rates were as follows: DMSO, $10.87 \pm 6.58 \%$; paclitaxel, $50.05 \pm 11.33 \%$; cisplatin, $41.88 \pm 8.55 \%$; anlotinib, $51.05 \pm 13.74 \%$; sunitinib, $30.03 \pm 12.85 \%$; and apatinib, $25.06 \pm 16.37 \%$ (Figure 1B). Because drug inhibition rate $>30 \%$ was regarded as an indicator of drug sensitivity, anlotinib treatment presumably resulted in significant tumor suppression in vitro.

\section{Anti-Tumor Effects of Anlotinib in vivo}

Given the in vitro data, nine PDXs (numbered as P0) were established. Three PDX models were selected and passaged to P3 by the same method, then used for in vivo experiments. Mice bearing the PDXs were treated with drugs for 21 days, at which time the 


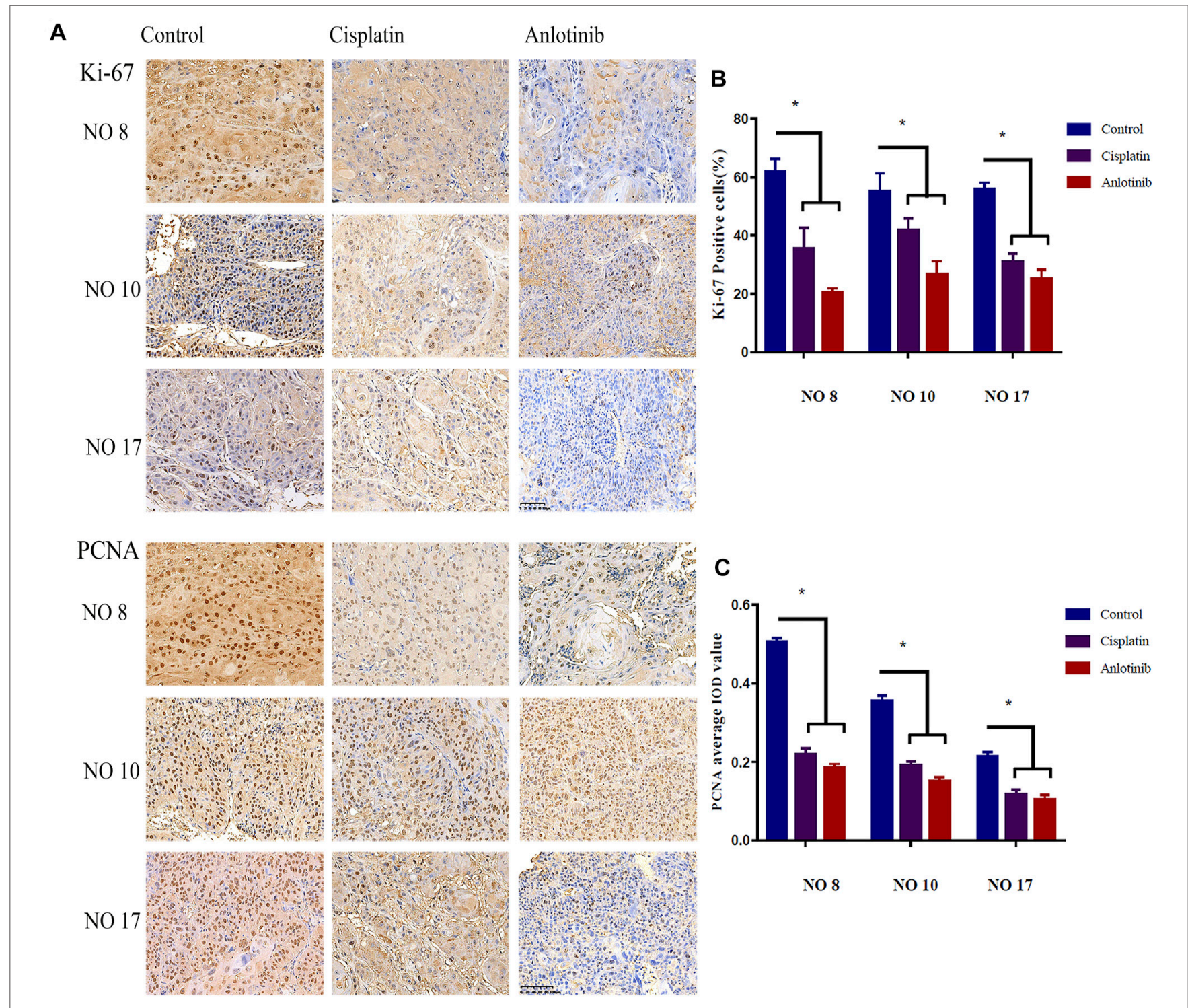

FIGURE 3 | (A) Representative image of immunohistochemical staining for Ki-67 and PCNA in mouse tumor tissues (scale bar: 20 um). Nuclear Ki-67, as well as nuclear and cytoplasmic PCNA, are stained clay red or brown. Percentages of cells expressing Ki-67 (B) and PCNA average IOD (C) in the various groups ( $\left.{ }^{\star}, p<0.05\right)$.

tumor volume in the control groups reached $1,500 \mathrm{~mm}^{3}$. The mice were then sacrificed. No mouse lost more than $10 \%$ of its body weight during the experiment, and no abnormal behavior was noted. Anlotinib was significantly more inhibitory (TGI 79.02\%) than cisplatin (TGI 40.57\%) (Figure 2A). The tumor volume in the anlotinib group on day 21 did not differ significantly from the initial volume (Figure 2B). The tumors in the anlotinib group were smooth-surfaced (unlike the tumors in the other two groups).

\section{Anlotinib Inhibits Tumor Cell Proliferation}

To evaluate the anti-tumor effects of anlotinib, the Ki-67 and PCNA indices of tumor sections were immunohistochemically evaluated. The Ki-67 and PCNA expression levels differed significantly among the three groups (Figure 3A). In the anlotinib group, the Ki-67 positive rates were $20.26 \pm 1.60 \%$ for patient no. $8,26.7 \pm 4.47 \%$ for patient no. 10 , and $25.13 \pm 3.24 \%$ for patient no. 17 ; these were significantly lower than the rates in the control group $(61.89 \pm 4.44 \%$ for patient no. $08,55.16 \pm 6.17 \%$ for patient no. 10 , and $55.85 \pm 2.23 \%$ for patient no.17; $p<0.05$ ) (Figure 3B). Similarly, compared to the control group, the PCNA-average integrated optical densities of $0.19 \pm 0.01,0.15 \pm 0.01$, and $0.10 \pm 0.01$ for patients no. 8,10 and 17 , respectively, were significantly lower than $(p<0.05)$ the corresponding figures in the control group $(0.50 \pm 0.01,0.35 \pm 0.01$, and $0.21 \pm 0.01$ ) (Figure 3C). The Ki-67-positivity rates and PCNAaverage integrated optical densities were lower in the anlotinib group than in the positive control group, suggesting that tumor growth was inhibited in HNSCC PDX mice; moreover, anlotinib significantly inhibited tumor cell proliferation. 


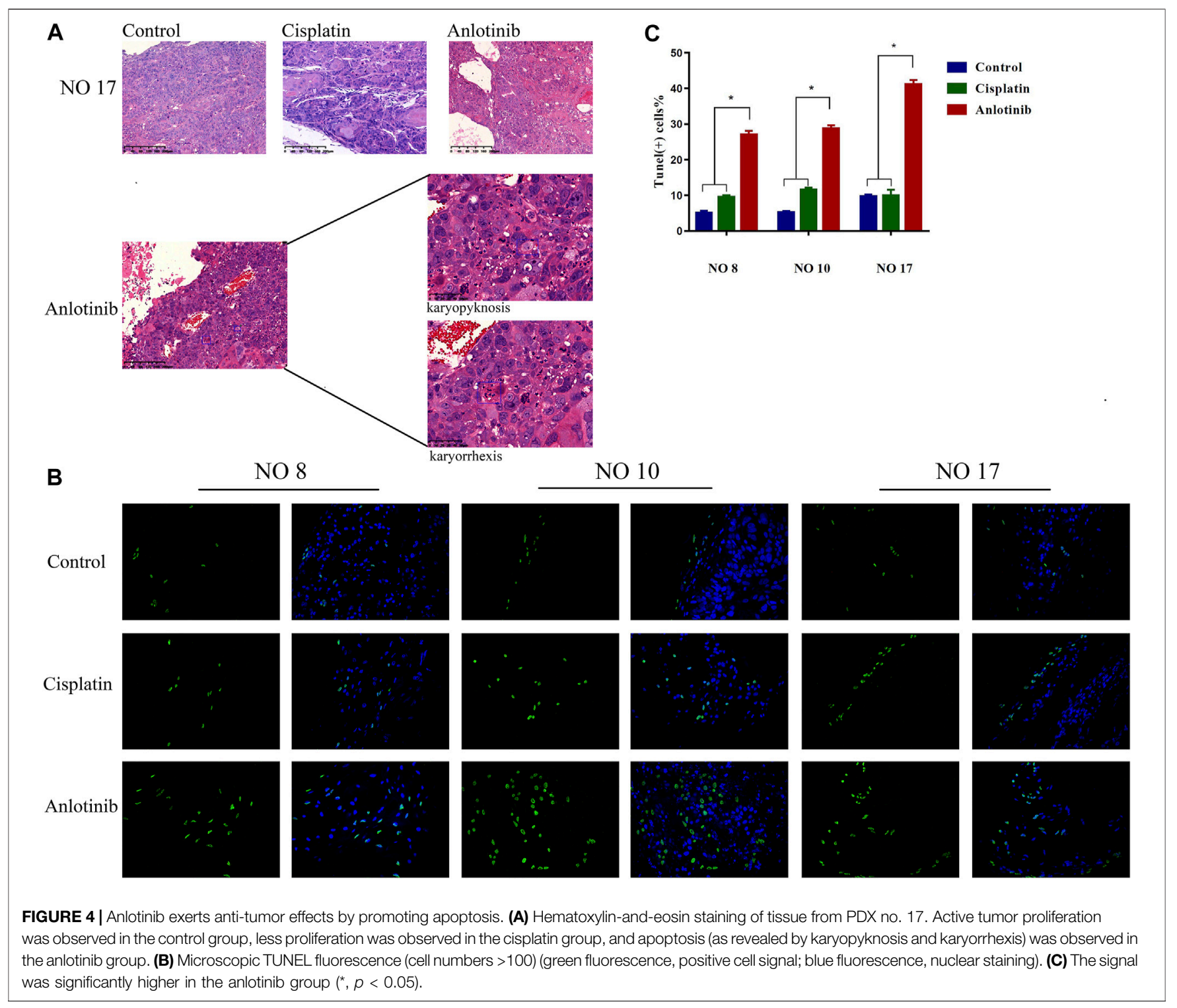

\section{Anlotinib Promotes HNSCC Tumor Cell Apoptosis}

Hematoxylin-and-eosin staining of the HNSCC tumor tissues of three tumor-bearing mice showed that the extents of necrosis and apoptosis varied among the groups. Tumors actively proliferated in the control group, but less so in the positive control and anlotinib groups. Apoptosis was maximal in the anlotinib group, in which karyopyknosis, karyorrhexis, and apoptotic bodies were observed (Figure 4A). TUNEL assays were performed, with green fluorescence indicating apoptosis and nuclei fluorescing blue. The highest apoptosis levels were observed in the anlotinib group (Figure 4B) (patient no. 8: $27.03 \pm 1.09 \%$, no. $10: 28.69 \pm 0.96 \%$, and no. $17: 41.12 \pm 1.21 \%$; the control values were no. $8: 5.01 \pm 0.63 \%$, no. $10: 5.245 \pm$ $0.33 \%$, and no. $17: 9.69 \pm 0.52 \%$; and the positive control values were no. $8: 9.50 \pm 0.58 \%$, no. $10: 11.50 \pm 0.71 \%$, and no. 17: $9.94 \pm 1.648 \%)(p<0.05)$ (Figure 4C). Therefore, anlotinib promoted tumor cell apoptosis.

\section{DISCUSSION}

HNSCC treatment remains challenging, although a multidisciplinary approach improves outcomes and increases survival. Local and distant metastases are the most important prognostic factors (22); several studies have shown that the expression of angiogenic factors and their receptors are closely related to the development, metastasis, and prognosis of HNSCC. Identifying drugs that suppress tumor development and metastasis is both important and difficult. Anti-angiogenesis inhibitors reduce tumor blood supply, create a low-oxygen microenvironment, and improve the efficacy of chemotherapy 
(23). Anlotinib is a small molecule, newly developed, oral and multi-targeting tyrosine kinase inhibitor that targets VEGFR1, VEGFR2/KDR, VEGFR3, c-Kit, PDGFR- $\alpha$, and the fibroblast growth factor receptors. Furthermore, it can inhibit both tumor angiogenesis and tumor cell proliferation (16, 24-26). Therefore, anlotinib anti-angiogenesis in combination with chemotherapy is a promising direction of research.

Although drugs targeting the vascular system effectively treat a variety of tumors, the therapeutic responses differ. Thus, selection of an appropriate drug is key in terms of personalized and precision medicine. Simple, individualized preclinical drug screening is important in this context. Most researchers of cancer biology use two- or three-dimensional models that have intrinsic limitations and may exhibit poor reproducibility. PDXs have been increasingly used for drug screening and evaluating drug resistance. PDXs retain tumor heterogeneity throughout implantation and in vivo culture. We subcutaneously implanted HNSCC tumor tissues into immunodeficient mice to establish PDXs for drug screening. Finally, nine PDXs (numbered as P0) were established. Three PDX models were selected and passaged to P3 by the same method, then used for in vivo experiments, because they were more efficient in developing tumors; moreover, their tumor sizes were similar. In vitro, anlotinib and paclitaxel suppressed tumors similarly. However, the individual results differed greatly. PDXs maintain the genetic characteristics of the parental tumors and recapitulate human tumor biology. They allow evaluation of the effects of different drugs in single patients and the effects of the same drug in different patients. Our average cisplatin tumor inhibition rate was $41.88 \pm 8.55 \%$ in vitro, and the TGI was $40.57 \%$ in vivo. Thus, the in vivo and in vitro data were consistent. However, the anlotinib inhibition rates differed significantly in vivo and in vitro; the former rate was higher. Hence, when using PDXs, it is important to consider drug absorption and metabolism.

Previous studies found that the expression of VEGF, FGFR1-4, and their receptors markedly affected tumor aggressiveness and prognosis; however, little research on how this affects HNSCC has been published $(27,28)$. Angiogenesis is very important in terms of tumor growth and metastasis; accordingly, anti-angiogenic agents are major components of current tumor treatments $(29,30)$. We found that anlotinib exhibited a better anti-tumor effect than did cisplatin. The tumor volumes in the anlotinib group did not increase significantly from the initial volumes. This allows a new clinical strategy. It is essential to improve the prognosis of patients with advanced HNSCC.

Of the many vascular factors, VEGF and its receptor VEGFR play key roles in tumor angiogenesis. Binding of VEGF to the receptor triggers receptor phosphorylation and the activation of downstream signaling by STAT3, Akt, and Erk-1/2, followed by endothelial cell proliferation, migration, and tube and microvessel formation $(31,32)$. Anlotinib targets JAK2/STAT3/VEGFR signaling, and the inhibition of angiogenesis halts tumor proliferation and promotes apoptosis (16, $17,33-35)$. Anlotinib is a potent tyrosine kinase inhibitor that inhibits VEGFR2 phosphorylation as well as that of PDGFR $\alpha / \beta$, c-Kit, RET, Aurora-B, C-FMS, and the discoid region of DDR1 $(14,15)$. Although anlotinib binds to both VEGFRs and other targets, the drug is more selective for VEGF family members (especially VEGFR2 and VEGFR3) than is sunitinib. Anlotinib selectively inhibits
VEGFR-dependent tumor cell proliferation, as described above. We confirmed that anlotinib suppressed HNSCCs in vivo, as revealed by the Ki-67 and PCNA immunohistochemical data. Compared to cisplatin (the positive control), anlotinib exhibited a greater inhibitory effect. Anlotinib also promoted tumor apoptosis.

Our results indicate the potential for anlotinib-sensitive HNSCC tumors. However, three PDX models were involved in in vitro experiments; other tumors may be resistant to anlotinib. More studies are needed to reveal the possible reasons. In addition, studies are needed to explore the mechanisms and determine the appropriate selection method to identify patients who may benefit from a treatment that includes anlotinib.

\section{CONCLUSION}

We successfully established a PDX model of HNSCC. To our knowledge, this is the first study to show the role of anlotinib in HNSCC in vivo. Anlotinib exhibited good anti-tumor activity in vitro and in vivo, inhibiting cell proliferation and promoting apoptosis. These findings suggest that anlotinib could be used for the treatment of patients with HNSCC.

\section{DATA AVAILABILITY STATEMENT}

The original contributions presented in the study are included in the article/Supplementary Material, further inquiries can be directed to the corresponding authors.

\section{ETHICS STATEMENT}

The studies involving human participants were reviewed and approved by First Affiliated Hospital of Nanchang University Ethics Committee (No. 2019017). The patients/participants provided their written informed consent to participate in this study. The animal study was reviewed and approved by the Institutional Animal Care and Use Committees of Nanchang University (RYE2019101905). Written informed consent was obtained from the individual(s) for the publication of any potentially identifiable images or data included in this article.

\section{AUTHOR CONTRIBUTIONS}

All authors listed have made a substantial, direct, and intellectual contribution to the work and approved it for publication.

\section{FUNDING}

This study was supported in part by grants from Natural Science Foundation of Jiangxi Province (2020BABL206066 to YX and 20192BAB205052 to WZ); Health and Family Planning Commission of Jiangxi Province Plan Project (20195120, 20203229). All sources of funding received for the research being submitted. 


\section{CONFLICT OF INTEREST}

The authors declare that the research was conducted in the absence of any commercial or financial relationships that could be construed as a potential conflict of interest.

\section{REFERENCES}

1. Bray F, Ferlay J, Soerjomataram I, Siegel RL, Torre LA, Jemal A. Global Cancer Statistics 2018: GLOBOCAN Estimates of Incidence and Mortality Worldwide for 36 Cancers in 185 Countries. CA: a Cancer J clinicians (2018) 68(6): 394-424. doi:10.3322/caac.21492

2. Vigneswaran N, Williams MD. Epidemiologic Trends in Head and Neck Cancer and Aids in Diagnosis. Oral Maxill Surg Clin North America (2014) 26(2):123-41. doi:10.1016/j.coms.2014.01.001

3. Bray I, Brennan P, Boffetta P. Projections of Alcohol- and TobaccoRelated Cancer Mortality in Central Europe. Int J Cancer (2000) 87(1): 122-8. doi:10.1002/1097-0215(20000701)87:1<122:aid-ijc18>3.0.co; $2-\mathrm{w}$

4. Ghantous Y, Schussel JL, Brait M. Tobacco and Alcohol-Induced Epigenetic Changes in Oral Carcinoma. Curr Opin Oncol (2018) 30(3):152-8. doi:10.1097/CCO.0000000000000444

5. Tribius S, Würdemann N, Laban S, Hoffmann TK, Sharma SJ, Klussmann JP. Update zu HPV-assoziierten Kopf-Hals-Karzinomen - Highlights der ASCOJahrestagung 2019. Hno (2019) 67(12):912-7. doi:10.1007/s00106-01900766-3

6. Allen CT, Law JH, Dunn GP, Uppaluri R. Emerging Insights into Head and Neck Cancer Metastasis. Head Neck (2013) 35(11):1669-78. doi:10.1002/ hed.23202

7. Pignon J-P, Maitre Al., Maillard E, Bourhis J, Group M-NC. Meta-analysis of Chemotherapy in Head and Neck Cancer (MACH-NC): an Update on 93 Randomised Trials and 17,346 Patients. Radiother Oncol (2009) 92(1):4-14. doi:10.1016/j.radonc.2009.04.014

8. Taurin S, Yang C-H, Reyes M, Cho S, Coombs DM, Jarboe EA, et al. Endometrial Cancers Harboring Mutated Fibroblast Growth Factor Receptor 2 Protein Are Successfully Treated with a New Small Tyrosine Kinase Inhibitor in an Orthotopic Mouse Model. Int J Gynecol Cancer (2018) 28(1):152-60. doi:10.1097/ igc.0000000000001129

9. Jayson GC, Kerbel R, Ellis LM, Harris AL. Antiangiogenic Therapy in Oncology: Current Status and Future Directions. The Lancet (2016) 388(10043):518-29. doi:10.1016/s0140-6736(15)01088-0

10. El-Kenawi AE, El-Remessy AB. Angiogenesis Inhibitors in Cancer Therapy: Mechanistic Perspective on Classification and Treatment Rationales. Br J Pharmacol (2013) 170(4):712-29. doi:10.1111/ bph.12344

11. Shweiki D, Itin A, Soffer D, Keshet E. Vascular Endothelial Growth Factor Induced by Hypoxia May Mediate Hypoxia-Initiated Angiogenesis. Nature (1992) 359(6398):843-5. doi:10.1038/359843a0

12. Kim KJ, Li B, Winer J, Armanini M, Gillett N, Phillips HS, et al. Inhibition of Vascular Endothelial Growth Factor-Induced Angiogenesis Suppresses Tumour Growth In Vivo. Nature (1993) 362(6423):841-4. doi:10.1038/ 362841a0

13. Ferrara N, Gerber H-P, LeCouter J. The Biology of VEGF and its Receptors. Nat Med (2003) 9(6):669-76. doi:10.1038/nm0603-669

14. Wang C, Chen J, Cao W, Sun L, Sun H, Liu Y. Aurora-B and HDAC Synergistically Regulate Survival and Proliferation of Lymphoma Cell via AKT, mTOR and Notch Pathways. Eur J Pharmacol (2016) 779:1-7. doi:10.1016/j.ejphar.2015.11.049

15. Sun Y, Niu W, Du F, Du C, Li S, Wang J, et al. Safety, Pharmacokinetics, and Antitumor Properties of Anlotinib, an Oral Multi-Target Tyrosine Kinase Inhibitor, in Patients with Advanced Refractory Solid Tumors. J Hematol Oncol (2016) 9(1):105. doi:10.1186/s13045-016-0332-8

\section{ACKNOWLEDGMENTS}

The English in this document has been checked by at least two professional editors, both native speakers of English. For a certificate, please see: http://www.textcheck.com/certificate/Xus4be.

16. Xie C, Wan X, Quan H, Zheng M, Fu L, Li Y, et al. Preclinical Characterization of Anlotinib, a Highly Potent and Selective Vascular Endothelial Growth Factor Receptor-2 Inhibitor. Cancer Sci (2018) 109(4):1207-19. doi:10.1111/ cas. 13536

17. Lin B, Song X, Yang D, Bai D, Yao Y, Lu N. Anlotinib Inhibits Angiogenesis via Suppressing the Activation of VEGFR2, PDGFR $\beta$ and FGFR1. Gene (2018) 654:77-86. doi:10.1016/j.gene.2018.02.026

18. Syed YY. Anlotinib: First Global Approval. Drugs (2018) 78(10):1057-62. doi:10.1007/s40265-018-0939-X

19. Karamboulas C, Meens J, Ailles L. Establishment and Use of PatientDerived Xenograft Models for Drug Testing in Head and Neck Squamous Cell Carcinoma. STAR Protoc (2020) 1(1):100024. doi:10.1016/ j.xpro.2020.100024

20. Loo DT. TUNEL Assay: An Overview of Techniques. Methods Mol Biol (2002) 203:21-30. doi:10.1385/1-59259-179-5:21

21. Lang L, Xiong Y, Prieto-Dominguez N, Loveless R, Jensen C, Shay C, et al. FGF19/FGFR4 Signaling axis Confines and Switches the Role of Melatonin in Head and Neck Cancer Metastasis. J Exp Clin Cancer Res (2021) 40(1):93. doi:10.1186/s13046-021-01888-9

22. Huang L, David O, Cabay RJ, Valyi-Nagy K, Macias V, Zhong R, et al. Molecular Classification of Lymph Node Metastases Subtypes Predict for Survival in Head and Neck Cancer. Clin Cancer Res (2019) 25(6):1795-808. doi:10.1158/1078-0432.CCR-18-1884

23. Ramjiawan RR, Griffioen AW, Duda DG. Anti-angiogenesis for Cancer Revisited: Is There a Role for Combinations with Immunotherapy?. Angiogenesis (2017) 20(2):185-204. doi:10.1007/s10456-017-9552-y

24. Tian S, Quan H, Xie C, Guo H, Lü F, Xu Y, et al. YN968D1 Is a Novel and Selective Inhibitor of Vascular Endothelial Growth Factor Receptor-2 Tyrosine Kinase with Potent Activity In Vitro and In Vivo. Cancer Sci (2011) 102(7):1374-80. doi:10.1111/j.1349-7006.2011.01939.x

25. Shen G, Zheng F, Ren D, Du F, Dong Q, Wang Z, et al. Anlotinib: a Novel Multi-Targeting Tyrosine Kinase Inhibitor in Clinical Development. J Hematol Oncol (2018) 11(1):120. doi:10.1186/s13045-018-0664-7

26. Lin B, Song X, Yang D, Bai D, Yao Y, Lu N. Anlotinib Inhibits Angiogenesis via Suppressing the Activation of VEGFR2, PDGFR $\beta$ and FGFR1. Gene (2018) 654:77-86. doi:10.1016/j.gene.2018.02.026

27. Gao L, Lang L, Zhao X, Shay C, Shull AY, Teng Y. FGF19 Amplification Reveals an Oncogenic Dependency upon Autocrine FGF19/FGFR4 Signaling in Head and Neck Squamous Cell Carcinoma. Oncogene (2019) 38(13): 2394-404. doi:10.1038/s41388-018-0591-7

28. Cohen EE, Davis DW, Karrison TG, Seiwert TY, Wong SJ, Nattam S, et al. Erlotinib and Bevacizumab in Patients with Recurrent or Metastatic Squamous-Cell Carcinoma of the Head and Neck: a Phase I/II Study. Lancet Oncol (2009) 10(3):247-57. doi:10.1016/s14702045(09)70002-6

29. Folkman J. Antiangiogenesis in Cancer Therapy-Endostatin and its Mechanisms of Action. Exp Cel Res (2006) 312(5):594-607. doi:10.1016/ j.yexcr.2005.11.015

30. Li J, Qin S, Xu J, Xiong J, Wu C, Bai Y, et al. Randomized, Double-Blind, Placebo-Controlled Phase III Trial of Apatinib in Patients with Chemotherapy-Refractory Advanced or Metastatic Adenocarcinoma of the Stomach or Gastroesophageal Junction. Jco (2016) 34(13):1448-54. doi:10.1200/jco.2015.63.5995

31. Olsson A-K, Dimberg A, Kreuger J, Claesson-Welsh L. VEGF Receptor Signalling ? in Control of Vascular Function. Nat Rev Mol Cel Biol (2006) 7(5):359-71. doi:10.1038/nrm1911

32. Siveen KS, Prabhu K, Krishnankutty R, Kuttikrishnan S, Tsakou M, Alali FQ, et al. Vascular Endothelial Growth Factor (VEGF) Signaling in Tumour 
Vascularization: Potential and Challenges. Curr Vasc Pharmacol (2017) 15(4): 339-51. doi:10.2174/1570161115666170105124038

33. Liang L, Hui K, Hu C, Wen Y, Yang S, Zhu P, et al. Autophagy Inhibition Potentiates the Anti-angiogenic Property of Multikinase Inhibitor Anlotinib through JAK2/STAT3/VEGFA Signaling in Non-small Cell Lung Cancer Cells. J Exp Clin Cancer Res (2019) 38(1):71. doi:10.1186/ s13046-019-1093-3

34. Wang G, Sun M, Jiang Y, Zhang T, Sun W, Wang H, et al. Anlotinib, a Novel Small Molecular Tyrosine Kinase Inhibitor, Suppresses Growth and Metastasis via Dual Blockade of VEGFR2 and MET in Osteosarcoma. Int J Cancer (2019) 145(4):979-93. doi:10.1002/ijc.32180
35. Ruan X, Shi X, Dong Q, Yu Y, Hou X, Song X, et al. Antitumor Effects of Anlotinib in Thyroid Cancer. Endocrine-related cancer (2019) 26(1):153-64. doi:10.1530/erc-17-0558

Copyright $\odot 2021 \mathrm{Hu}, \mathrm{Guo}, \mathrm{Yu}, \mathrm{Dai}$, Xiong, He and Zhou. This is an open-access article distributed under the terms of the Creative Commons Attribution License (CC $B Y)$. The use, distribution or reproduction in other forums is permitted, provided the original author(s) and the copyright owner(s) are credited and that the original publication in this journal is cited, in accordance with accepted academic practice. No use, distribution or reproduction is permitted which does not comply with these terms. 